\title{
Tool wear monitoring in roughing and finishing processes based on machine internal data
}

\author{
Tiandong Xi ${ }^{1}$ (D) . Igor Medeiros Benincá ${ }^{1} \cdot$ Sebastian Kehne ${ }^{1} \cdot$ Marcel Fey $^{1} \cdot$ Christian Brecher $^{1}$
}

Received: 16 November 2020 / Accepted: 1 February 2021 / Published online: 5 March 2021

(C) The Author(s) 2021

\begin{abstract}
Data analytics plays a significant role in the realization of Industry 4.0. By generating context-related persistent datasets, every manufacturing process in real production becomes an experiment. The vision of Internet of Production (IoP) is to enable real-time diagnosis and prediction in smart productions by acquiring datasets seamlessly from different data silos. This requires interdisciplinary collaboration and domain-specific expertise. In this paper, we present a novel tool wear monitoring system for milling process developed in the context of IoP. This system is based on high-frequency data from the numerical control of the production machine without additional sensors. The novelty of this paper lies in the introduction of virtual workpiece quality and fusion of multiple build-in sensor signals and a force model as decision support. This bridges the time gap between quality inspection and production at the shop floor level, establishes an automated statistical process control system, and provides a more plausible prediction of tool lifetime. The monitoring of two different milling processes in a real production environment is exemplary demonstrated in this paper. The first case is a face roughing process with the aim of rapidly removing large amounts of material. The second case is a face finishing operation that follows roughing and aims to achieve the desired surface quality.
\end{abstract}

Keywords Tool wear · Digital twin · Data analytics · Quality inspection · Condition monitoring

\section{Introduction}

Modern production is characterized by vast amounts of data in numerous domain-silos, which are neither easily accessible, interpretable, nor connected to gain knowledge [1]. However, these data and associated analytics have big potentials in terms of improving the product and production quality. The concept of Internet of Production (IoP) targets collecting and processing context-based data from production and realizing real-time control of production processes [2]. One challenge is to establish a stable network infrastructure to be able to collect and handle vast amounts of production data in real-time. Hard- and software components for such infrastructure in machine tool industry are introduced in [3] and serve as background for further analyses in this paper. Another major challenge

Tiandong Xi

t.xi@wzl.rwth-aachen.de

1 Laboratory for Machine Tools and Production Engineering, (WZL) of RWTH Aachen University, Steinbachstr. 19, 52074, Aachen, Germany is to develop fast computing models that are accurate and plausible enough to help to improve the production processes. These models are driven by real-time production data, thus providing deeper insights than a priori simulation models. For example, based on high-frequency machine internal data, a digital twin of being manufactured part can be generated by material removal simulation, which further approximates the workpiece quality and enables a quick quality feedback loop [4, 5]. Statistical process control has been widely used in the manufacturing and process industries to monitor the performance of a process over time and eliminate quality problems $[6,7]$. In this paper, we present a novel tool wear monitoring system developed in the context of IoP. The novelty of this research lies in the introduction of virtual workpiece quality and fusion of multiple standard built-in sensor signals as decision support for cutting processes with different requirements, i.e., for roughing processes, tool lifetime can be extended to its wear limit since surface quality is not the focus point. While for fine finishing processes, even mild wear could cause unwanted quality issues since here the surface quality is decisive. All experiments in this study were realized with real production, not laboratory experiments, which shows 
the flexibility and adaptability of the suggested approaches when facing the industrial environment.

This paper is organized as follows: Following this "Introduction" section, Section 2 reviews the background and existing methods for cutting tool monitoring. The models used in this paper, such as the force model based on machine internal data and a dexel-model for cutting simulation are briefly introduced in Section 3. Subsequently, two exemplary use cases in the industry are demonstrated in Section 4. Two different approaches are introduced and discussed in detail. One is based on a single process quality indicator and another is based on a color map representation of the process. Conclusions and outlooks are provided in the last section.

\section{State of the art: Tool wear monitoring}

The diagnosis and prognosis of the optimal moment for tool change are essential for automated mass production. If tools are changed too often and too early, machine-idle time, thus material and production cost, will increase. However, a too late replacement timing may cause quality issues or even unscheduled machine downtime due to tool breakage. Normally, cutting tools are replaced regularly after a fixed number of manufactured parts. This decision is made by experienced machine operators. Due to the complexity of milling process and numerous random influences before and during manufacturing, the tool changing timing tends to be very conservative. Different tool condition monitoring systems have been developed to expose this hidden potential. Two types of monitoring methods can be distinguished: direct monitoring and indirect monitoring [8].

As its name suggests, direct monitoring methods capture directly the edge of the cutting tools by using vision systems. The tools will be either took off and examined on a special measuring machine or directly measured in a machine tool using an integrated CCD camera and light systems [9]. The first approach has the most precise outcome, but results in a long idle time in production. The latter solution can be seen in some modern machine tools, such as a61nx machining center from Japanese machine tool manufacturer Makino. The monitoring system is capable of effectively detecting tool breakage by comparing images before and after cutting. However, when it comes to monitoring flank wear or crater wear, the accuracy of image processing systems is easily affected by coolants and metal chips that are left on the tool surface [8].

In indirect monitoring approaches, tool condition is estimated by analyzing one or more signals from different sensors that are related to wear and tear. Typical signals are cutting forces, motor currents, acoustics, and tool vibrations. Cutting force has been selected as a reliably indicator for tool monitoring in [10] due to its fast response time and high sensitivity. Different signal processing strategies and post-processing techniques can be adopted to enhance the performance of force-based wear monitoring systems [11]. However, additional dynamometers need to be installed on the machine table or to the main spindle, which is very difficult in industrial applications. Apart from that, high-precision force sensors are very expensive and often lead to a reduction of local machine stiffness. To overcome this paradox, smart cutting tools for dry turning process are introduced in $[12,13]$, which utilize surface acoustic wave sensors or piezoelectric films to measure the cutting force.

Besides additional sensors, estimation methods based on tracking coefficients of analytical force models are introduced in $[14,15]$, where knowledge of the cut geometry is required in the first place to estimate the model coefficients. Another very often used signal is motor current, as it correlates to process force. One big advantage of using current signal is that further modification at machine tool is not necessary. Current signals of the servo motors can be acquired through special interfaces in modern machine tools with little effort. A tool-condition criterion based on the measured current values of the main spindle and drive motors is introduced in [16]. The proposed method shows a good capability of monitoring the tool condition for different cutting parameters. However, current signals are greatly influenced by friction in feed drive systems, viscous damping of mechanical components, and torque ripple of servo motors $[17,18]$. Tool monitoring systems based on vibration signals can also be often found in many literature works. Accelerometers are mounted either on the spindle shaft [19] or on the workpiece [20]. The collected signals are then processed in time or/and frequency domain in order to extract features and estimate tool condition based on training samples. Nevertheless, vibration signals are very sensitive and can be error prone due to noise, such as the influence of coolant and non-cutting vibrations [8]. As shown above, using only a single sensor for tool monitoring is limited in some cases. Therefore, multiple sensors and signals are usually adopted in sense of sensor fusion to eliminate the uncertainty and achieve a robust prediction result [21]. A simple machine learning allied with datadriven methods for tool monitoring is described in [23]. The input data are spindle load, spindle power, and axis load, respectively, which are provided by the machine tool in $1 \mathrm{~Hz}$ through a standard Internet protocol. Experimental results show that predicting accuracy with multi-domain and multi-sensory signals (e.g., force, vibration, and current) is higher than that of other methods [22]. However, no reliable commercial solutions for tool and process monitoring can be found.

As a conclusion of the literature review, there is a variety of sensors and signals that can be used to monitor 


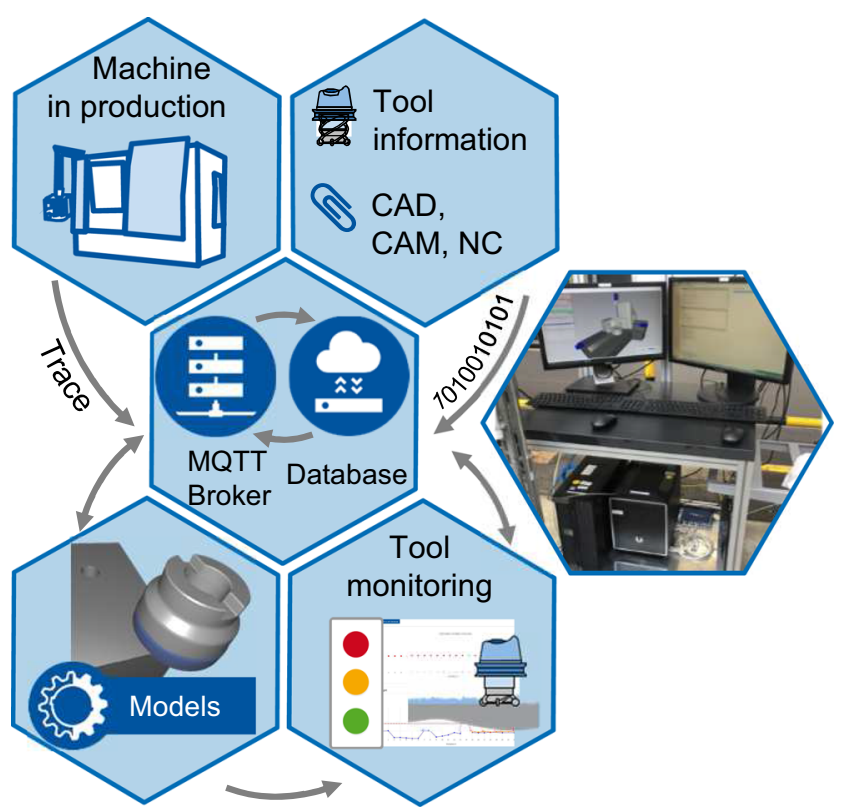

Fig. 1 System framework and data flow. Top: different data sources. Middle: middleware for data processing and data storage. Bottom: models to analyze data in real time

the tool wear and good results are expected in laboratory experiments. However, the question is to what extent these methods can be implemented at shop floor level. On the one hand, external sensors, such as force and vibrations sensors, are very difficult to integrate into a machine without affecting the existing manufacturing processes. On the other hand, a lot of experimental samples are required to train an accurate model, which will increase the implementation effort. This paper introduces a new concept for monitoring tool wear at shop floor level. The research was carried out by analyzing historical production data. To predict the optimal timing for tool changing, virtual quality of the workpiece was combined with multiple machine internal signals and a force model.

\section{Turning data into value}

The overview of the utilized system framework for data processing and tool monitoring is briefly described in Fig. 1. A customized software solution has been installed on an edge computer near the machine tool, which records continuously and automatically all the relevant NC-internal signals of the machine at a sampling rate of $500 \mathrm{~Hz}$ (Fig. 1, top). A list of the recorded high-frequency signals can be found in Table 1. In addition, event-based information such as tool number in spindle, active zero offset, name of the current $\mathrm{NC}$ program, and tool information were also recorded. All these data were transmitted via the MQTT protocol to a time series database [3] (Fig. 1,
Table 1 List of high-frequency trace signals

\begin{tabular}{lll}
\hline Signal & Description & Unit \\
\hline$T S$ & Unix timestamp & $\mathrm{ms}$ \\
$X C / Y C / Z C$ & Drive motor current & $\mathrm{A}$ \\
$X 1 / Y 1 / Z 1$ & Axis position from motor encoder & $\mathrm{mm}$ \\
$X 2 / Y 2 / Z 2$ & Axis position from linear encoder & $\mathrm{mm}$ \\
$L N$ & Line number of the current NC & - \\
$S C$ & Spindle current & A \\
$S S$ & Spindle speed & $\mathrm{rpm}$ \\
\hline
\end{tabular}

middle). Different real-time models and simulation software packages have been developed at Laboratory for Machine Tools and Production Engineering (WZL) to create a digital shadow of the machining process and final workpiece based on the collected trace data. These models are then used to realize the online tool wear monitoring (Fig. 1, bottom).

\subsection{Force model}

One of the most important factors that describes the cutting operation is the process force, which is difficult to measure in automated production. Force model based on motor current is a very common approach to estimate cutting forces in real-time [24]. However, the drive motor in the control loop acts as a low-pass filter, so that low- and high-frequency axis loads can not (exactly) be determined [25]. The force model used in this paper is based on the method described in [25]. Instead of using the drive motor current, the measured position values of the linear scale and motor encoder are taken into account. For a feed drive axis with two position measuring systems, e.g., an indirect measurement system $X 1$ and a direct measurement system $X 2$, the axis force $F_{x}$ is calculated as:

$F_{x}=\Delta x_{\text {corrected }} \times k_{x, p o s}(x)$

where $\Delta x_{\text {corrected }}$ is defined as:

$$
\begin{aligned}
\Delta x_{\text {corrected }}= & X 1-X 2+C_{f r i c}(v)+C_{p i t c h}(x) \\
& +C_{a c c}(a)+C_{d i r}(v)
\end{aligned}
$$

$C_{f r i c}(v), C_{p i t c h}(x), C_{a c c}(a)$, and $C_{d i r}(v)$ are the velocitydependent friction error, the position-dependent pitch error of the ball screw drive, the acceleration error, and the direction-dependent error, respectively. These errors were identified by reference runs in the first place and stored as lookup tables. The stiffness $k_{x \text {,pos }}(x)$ was experimentally determined by measuring the force applied to the tool center point and the changing of the machine internal data under static conditions at different axis positions. The obtained stiffness characteristics of motor current and difference between encoders are shown in Fig. 2. The difference 
Fig. 2 Measured stiffness characteristics. Left: force and difference between measurement systems. Right: force and motor current
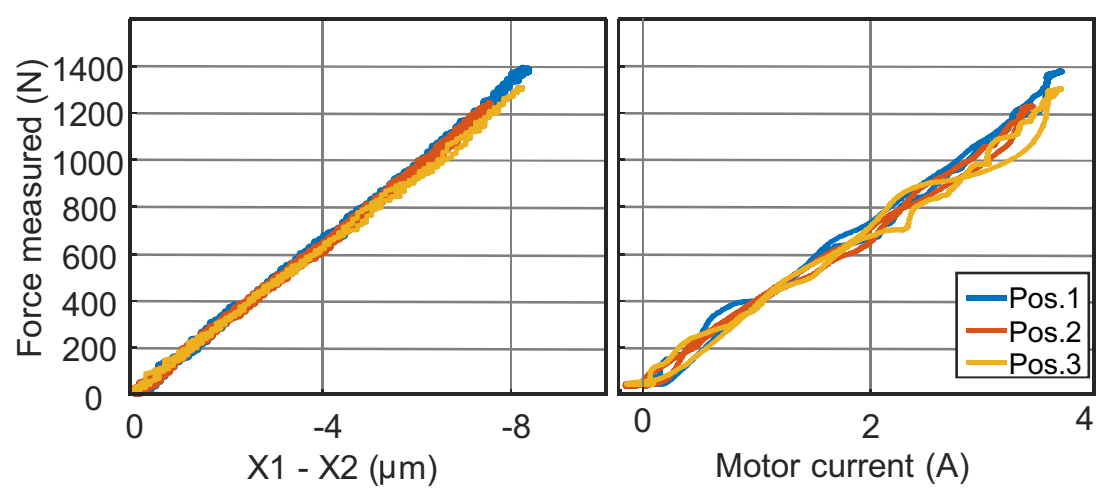

between encoder signals $(X 1-X 2)$ shows a better linearity and virtually position-independent behavior.

The developed force model was experimentally validated by cutting tests with a force measuring platform in the milling machine DMC-80-H-linear. The workpiece material was GG-25. The sampling rates of the force measuring platform and the force model were $10 \mathrm{kHz}$ and $500 \mathrm{~Hz}$, respectively. A comparison of the estimated force and measured force is given in Fig. 3. The estimated force shows an overall good agreement with the measured force, except with regard to the bandwidth, which is limited by the position regulation cycle of the $\mathrm{CNC}$ machine tool.

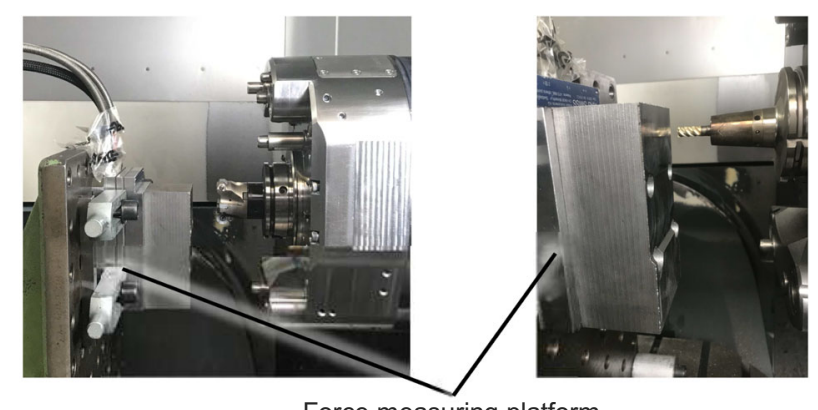

Force measuring platform
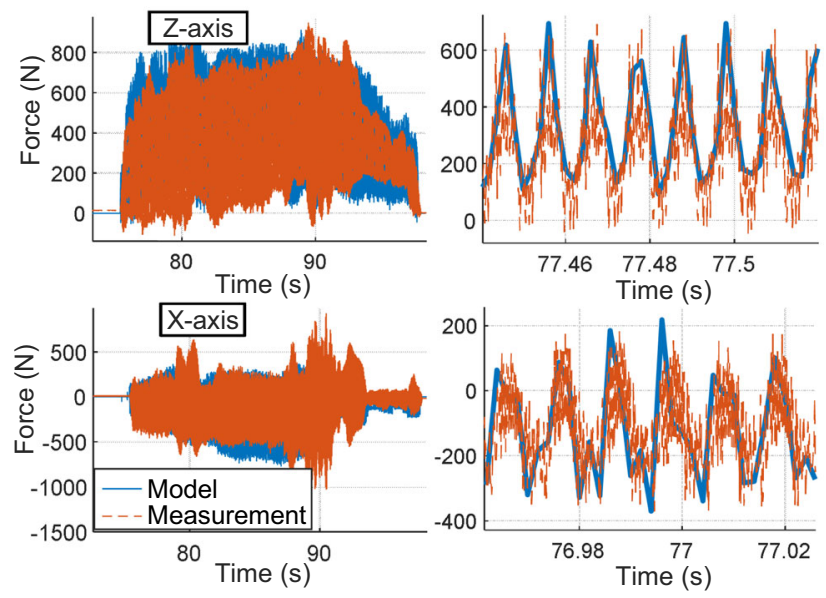

Fig. 3 Validation of the force model with force measuring platform

\subsection{Material removal simulation in real-time}

In manufacturing, quality control is usually done by regular quality checks. However, there is still a considerable time delay when feeding back negative trends or tolerance violations [4]. In order to shorten the time delay between the production and the subsequent quality measurement, a process-parallel chip removal simulation has been developed at WZL. Data from the CAD-CAM-NC process chain and the real production data are linked together. The actual tool center point (TCP) is calculated based on the axis position $X 2$ given by the $\mathrm{CNC}$ and the measured machine stiffness $k_{\text {machine }}(x)$ :

$X_{T C P}=X 2+F_{x} / k_{\text {machine }}(x)+x_{\text {geometric }}+x_{\text {thermo }}$

where the geometric error of the machine tool $x_{\text {geometric }}$ was identified in the first place. The influence of temperature $x_{\text {thermo }}$ is neglected here because the machine is in series production and the hall temperature is maintained at a constant level. The workpiece itself is modeled by a 3-dexel representation and the tool is modeled by a layered depth normal image representation, which can be transformed into a local dexel model when the tool engages the workpiece [4]. As a result, a digital twin of the being manufactured workpiece is generated in real-time, which includes the random effects in production. Figure 4 shows the interface of the real-time material removal simulation. The simulation was performed on a computer with an $15-7600 \mathrm{k}$ CPU and a GTX 1080 graphics card. As shown in the screenshots, similar tool marks can be seen on both real and virtual workpieces.

The generation of virtual workpiece further allows an automated virtual quality check. For example, for flatness measurement, the measurement path from a coordinate measurement machine (CMM) can be imported into the software developed in WZL called virtual measurement machine (VMM) [4] as point cloud (Fig. 5, top left). These points are then orthogonally projected onto the workpiece surface to extract the intersection points, which represents the raw surface geometry. Subsequently, a least square 
Fig. 4 Material removal simulation and comparison of real workpiece vs. virtual workpiece
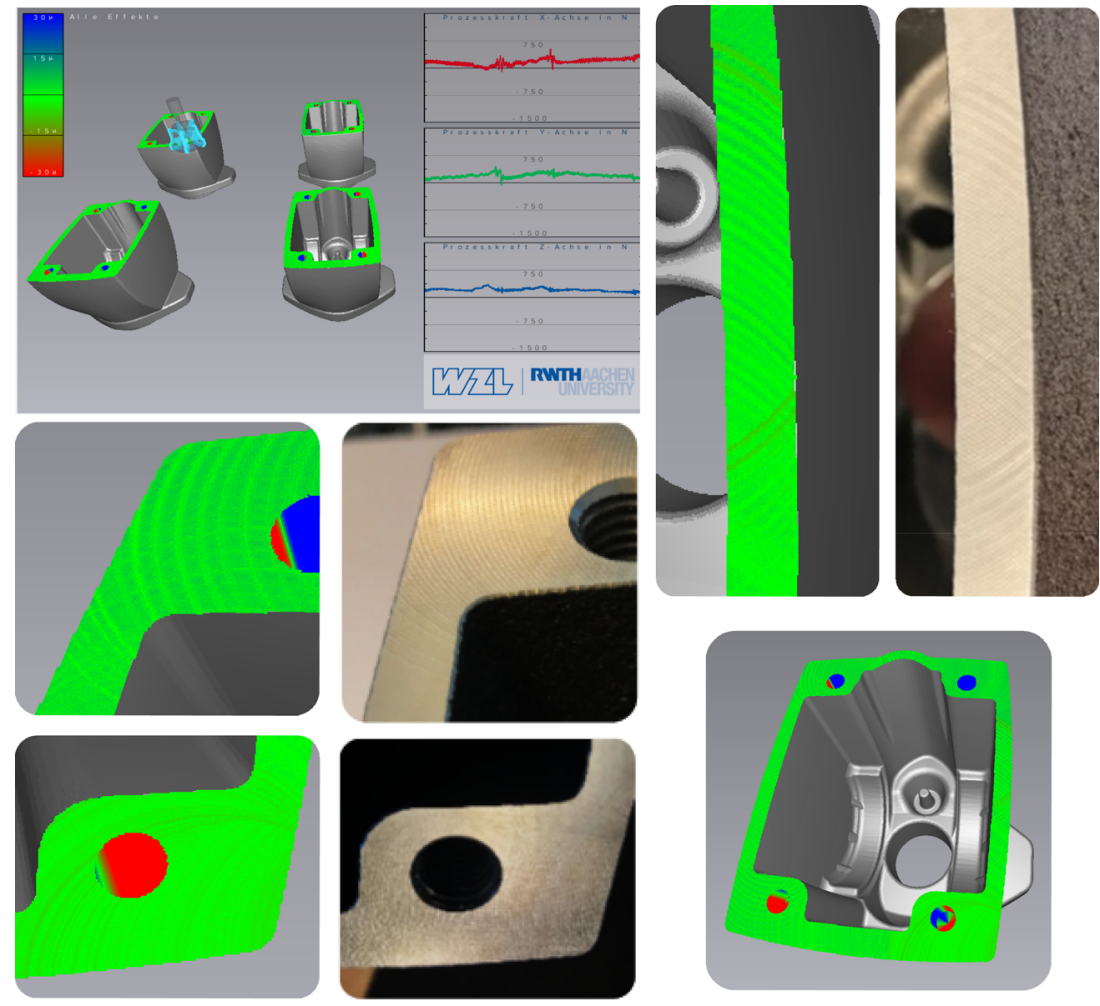

fitting is performed to find a fitted plane in 3-dimensional space to the raw data points. The distances from the raw data points to the fitted plane are then calculated and smoothed with a spline filter [26]. A comparison of the measurement report obtained from the virtual measurement and real measurement is given in Fig. 5. The flatness of the measured surface is defined as the difference between the maximum and minimum value of the filtered curve. In this case, the flatness of the virtual workpiece and real workpiece are $0.0082 \mathrm{~mm}$ and $0.008 \mathrm{~mm}$, respectively. Although the virtual measurement here shows good agreement with real measurement, virtual quality reports can never replace a high-precision measurement machine [27]. However, automated virtual quality measurement can enable a statistical quality control, give information about whether the process is stable or not, and provide indications whether the tool wear progress will affect the workpiece quality.

\section{Exemplary use case for tool wear monitoring}

\subsection{Description of the scenario}

Some parts of a raw casting need to be machined to meet the specific tolerance requirements. In this scenario, four raw casting parts were fixed in a clamping device and machined in a batch as shown in Fig. 4, top left. To achieve the desired surface quality ( $0.03 \mathrm{~mm}$ flatness), first, a roughing tool (hereinafter referred to as T1) was used to rapidly remove the raw casting material (see Fig. 6). Then, a finishing tool (hereinafter referred to as T2) was used to produce the final geometry. Both tools were replaced regularly after 20 batches, i.e., 80 workpieces in serial production. The main goal of this paper is to develop a tool monitoring system that optimizes the tool changing process by determining the actual tool condition. Meanwhile, the following problems have been encountered that may not appear in research environments:

- Data came directly from a production machine. Experiment on the machine was only possible to a limited degree without disturbing the normal production.

- Real production is complicated and fraught with uncertainty. For example, the coolant is turned on during cutting or the cutting volume is not always constant.

- Finishing follows roughing. The result of the roughing process could have an influence on the subsequent finishing process.

\subsection{Tool monitoring methods}

This section presents two different approaches to evaluate the tool wear condition considering the execution of finishing and roughing processes. Both developed tool 
Fig. 5 Comparison of real measurement (CMM) vs. virtual measurement (VMM)
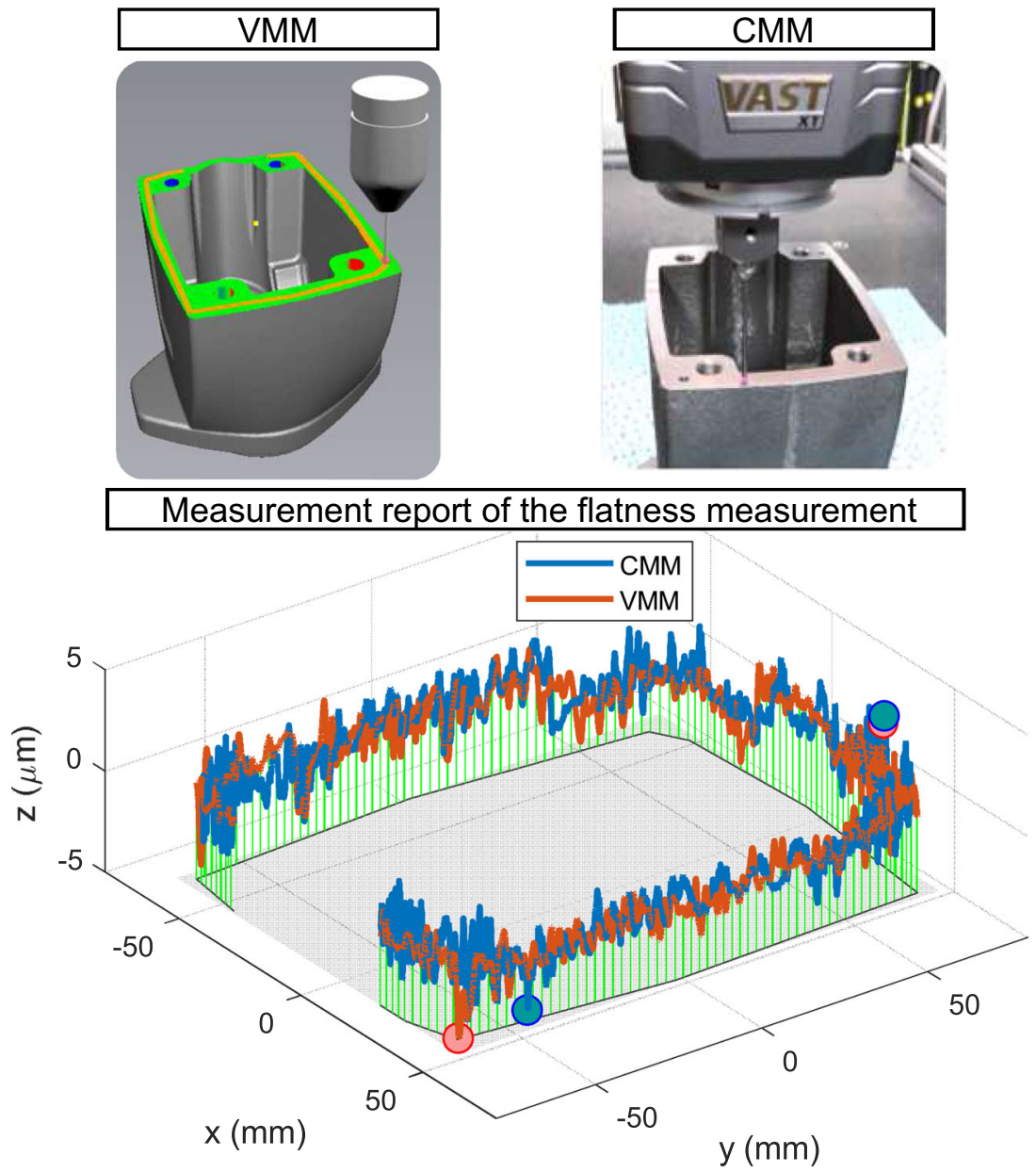

monitoring systems were based only on machine internal data. Both approaches used the information such as NC line number and tool number in main spindle; thereby, the corresponding production data with $\mathrm{T} 1$ and $\mathrm{T} 2$ were extracted from the data stream. The mean forces in $\mathrm{Z}$ direction over the entire roughing and finishing process were proved to be related to tool wear. As shown in Fig. 7, the mean force in $\mathrm{Z}$ direction increases with the number of manufactured workpieces. The moment of regular tool changing after around 80 workpieces can be clearly observed by the averaged force. Furthermore,

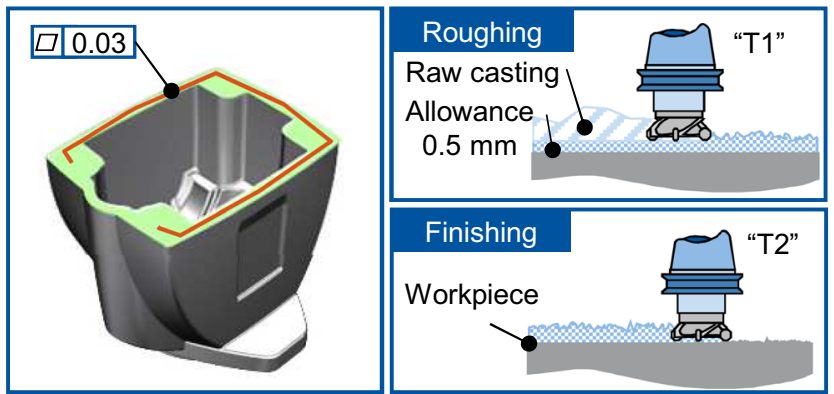

Fig. 6 Description of the scenario different combinations of roughing and finishing tools have been tested, such as $\mathrm{T} 1$ is nearly worn out while $\mathrm{T} 2$ is new or T1 is new while $\mathrm{T} 2$ has been used for a while. Results indicated that influence of the roughing tool on the finishing tool was negligibly small. The trend development of the finishing tool was not affected by roughing. Therefore, it is reasonable to consider these two processes separately in the following sections. Besides the mean force, similar trend development as in Fig. 7 were obtained by taking the

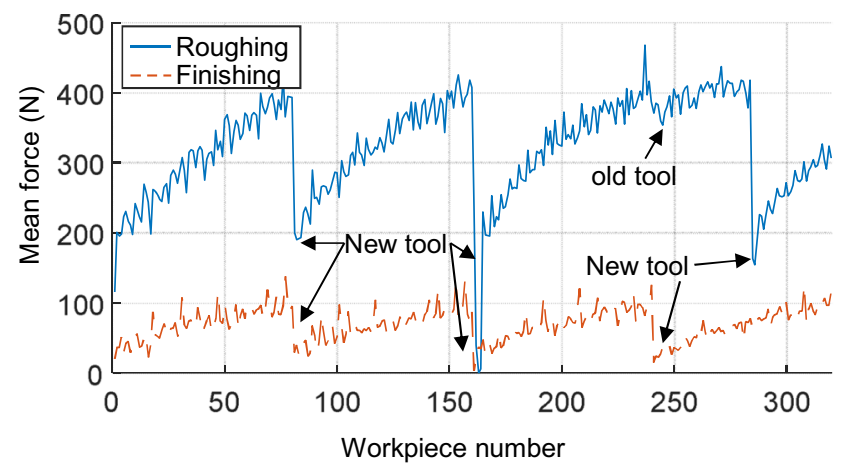

Fig. 7 Mean forces of the roughing and finishing process in $\mathrm{Z}$ direction 
average value of the main spindle current or the drive motor current.

\subsection{Monitoring of the roughing process}

Approach 1: the aim of roughing is to effectively remove large amounts of material. The surface quality does not matter, as it will be finished later. A roughing tool can be used as long as the cutters are not worn out and the process is stable. With this in mind, a process quality indicator (PQI) based on the cutting force and spindle current similar to [16] can be adopted as:

$$
P Q I=\sqrt{w_{1} \cdot\left(F_{Z}-F_{Z, r e f}\right)^{2}+w_{2} \cdot\left(I_{S P}-I_{S P, r e f}\right)^{2}}
$$

where $w_{1}$ and $w_{2}$ are weighting factors; $F_{Z}$ and $I_{S P}$ are the root mean square values of the cutting force in $\mathrm{Z}$ direction and spindle current, respectively. $F_{Z \text {,ref }}$ and $I_{S P \text {,ref }}$ are the reference force in $Z$ direction and the reference spindle current, respectively. Force $F_{Z}$ is estimated based on the difference between linear scale and motor encoder. The reason for using force instead of directly using drive current is that the encoder signals have a better linearity and disturbance forces can be corrected to achieve a better result (see Section 3.1). Figure 8 (left) shows the trend of the calculated PQI as a function of workpiece batch number. Note: every time, four workpieces were manufactured in one batch. A total of 600 workpieces (with 30 tools) from the historical data are plotted in the diagram. Due to some random effects of the cutting process, such as built-up-edge, raw casting form variation, there is a $\pm 15 \%$ variation in the calculated PQI. This indicator is mainly affected by tool wear, when all the process parameters remain unchanged. Based on the rising trend, an upper warning or control limit can be defined, which indicates a need for tool replacement. Figure 8 (right) shows two cases that were detected by this monitoring method. The cross markers already show a high PQI at the beginning and the indicator rises continuously with the number of workpieces. This indicates that instead of a new roughing tool, a heavily worn tool was changed in. The square markers have an outlier when manufacturing the first workpiece. The cutting force here was two times higher compared with the usual cutting processes. As expected and verified by the production documentation, this part had a quality issue. Possible reasons for the outlier could be the wrong tool length in tool table or the wrong zero point shift in NC program.

Approach 2: since PQI is a mean-value-based quality indicator, a second approach in time domain was investigated for the roughing process. Therefore, a tool wear indicator based on cutting force, spindle current, and unix timestamps is proposed. The indicator is based on a color map representation that illustrates the interpolated historical process behavior dependent on the cutting forces $F_{Z}$ and spindle current $I_{S P}$ over the machining time interval $t$. Thus, each combination of the point pair $\left(F_{Z}\right.$ and $\left.I_{S P}\right)$ is quantified with an absolute value of time, thus indirectly quantifying the time spent for each pair of force and current points acquired in the process. Figure 9 shows four color maps which characterize the progressive wear of the roughing tool from its beginning. It is notable that in the first figure with a new cutting tool, the process uses a small amount of current and has a larger cutting force range. However, with the increase of the manufactured workpieces, the process needs to consume more power and higher cutting forces to perform the same task as before. This is due to the fact that the tool is progressively wearing out. These characteristics can also be observed by the decrease in the effective area of the color map. Thus, there is a direct correlation of tool life with the size of the effective area on the color map.

\subsection{Monitoring of the finishing process}

Approach 1: the aim of the finishing process is to achieve the desired surface flatness of $30 \mu \mathrm{m}$. A $63-\mathrm{mm}$ face milling cutter with five inserts was used to remove the 0.5 $\mathrm{mm}$ allowance which was left after roughing. The cutting force in finishing was therefore very small (under $200 \mathrm{~N}$ ). Furthermore, due to the varying width of the surface being cut, using only mean values over the whole process as in Section 4.3 does not provide the best indicator. To enhance the estimation accuracy and reduce the variation of PQI, three signals were fused together to build the wear indicator (Fig. 10). Besides force and spindle current, the spindle speed was also found to be related to tool wear. As the tool wear progresses, it requires more power for cutting. Thus, when the tool engages the material for the first time, spindle speed decreases as the cutting force works as an external disturbance, which excites the speed control loop. The decrease of spindle speed is quite robust for tool wear monitoring in this case. Similar to Eq. 4, the process quality indicator based on these three signals was calculated.

As shown in Fig. 11, a good describable rising trend of PQI depending on the workpiece batch number is evident. However, it is unclear whether the tools have reached their maximum lifetime after 20 batches. Conventionally, a lot of time-consuming laboratory tests are required to identify the tool wear limit. Especially when it comes to monitoring the finishing tool, light to medium tool wear and tear could already lead to a bad surface finish, which is the decisive factor for finding an appropriate tool change timing. To overcome this problem, the virtual quality (see Section 3.2) was adopted to support the monitoring of the finishing tool. Historical data indicated that the finishing process and the workpiece quality were stable after 20 batches. Therefore, a 
Fig. 8 Process quality indicator for the roughing process. Left: historical data. Right: unusual cases
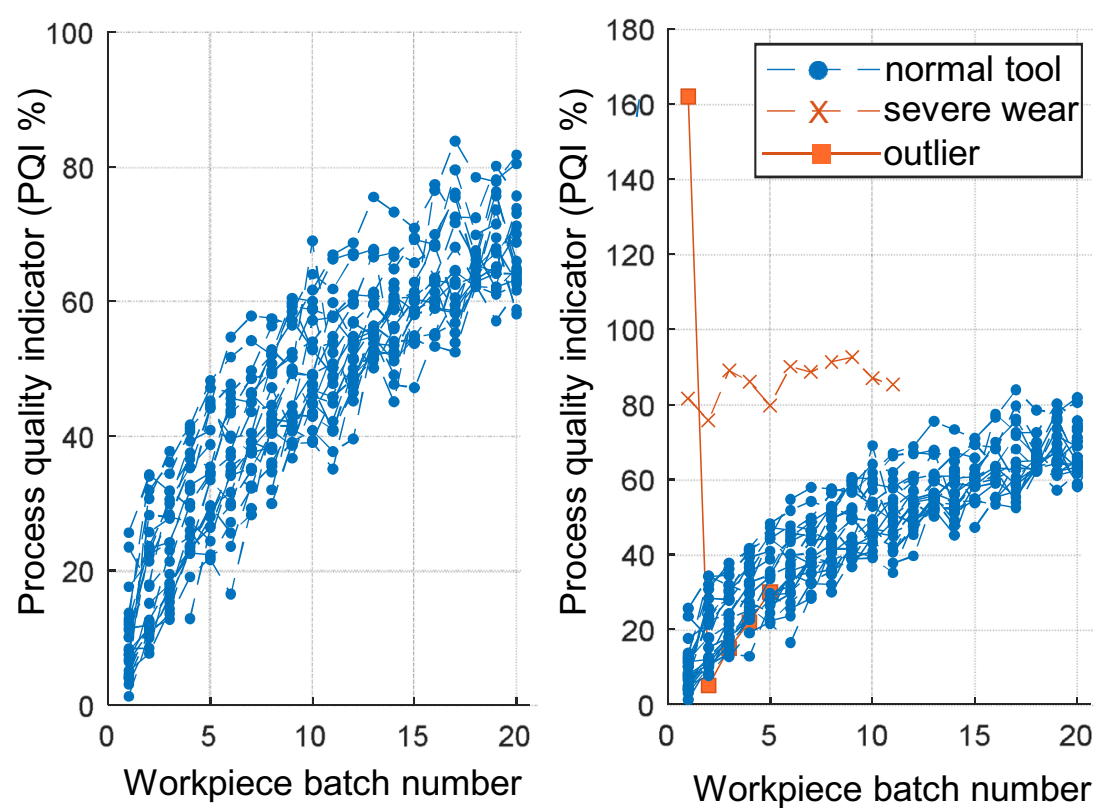

small test series has been conducted, where a finishing tool was carefully adopted for 25 batches or 100 workpieces. This corresponds to a $25 \%$ more lifetime compared with 20 batches. Every fourth workpiece was examined by a highprecision coordinate measuring machine. All workpieces were virtually manufactured and measured based on highfrequency trace data. As shown in Fig. 12, even though the PQI rises continuously after finishing 80 workpieces, which indicates higher wear and tear of the tool, the surface flatness quality is still maintained at a good level near 8 $\mu \mathrm{m}$. The $100 \%$ PQI in Fig. 12 is an approximated wornout limit. However, the wear limit was never reached in real production, as this would cause a high scrap rate. We assumed that the wear status is at $80 \%$ after 25 batches in the real production. In order to safely get close to the maximum lifetime of the tools, PQI was combined with virtual quality, which offers a statistically controlled production and a quick quality feedback. Nevertheless, certified real measurement
Fig. 9 Color maps representation of tool wear progression for the roughing process
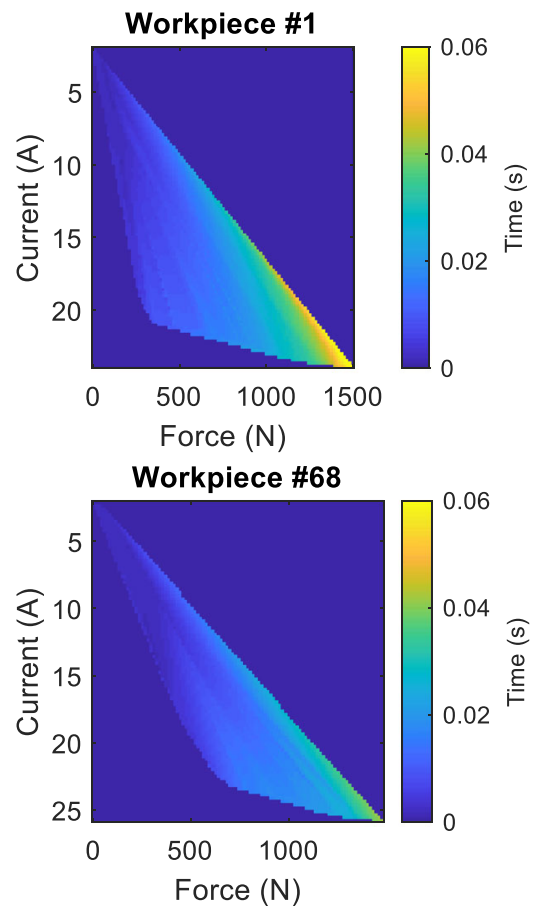
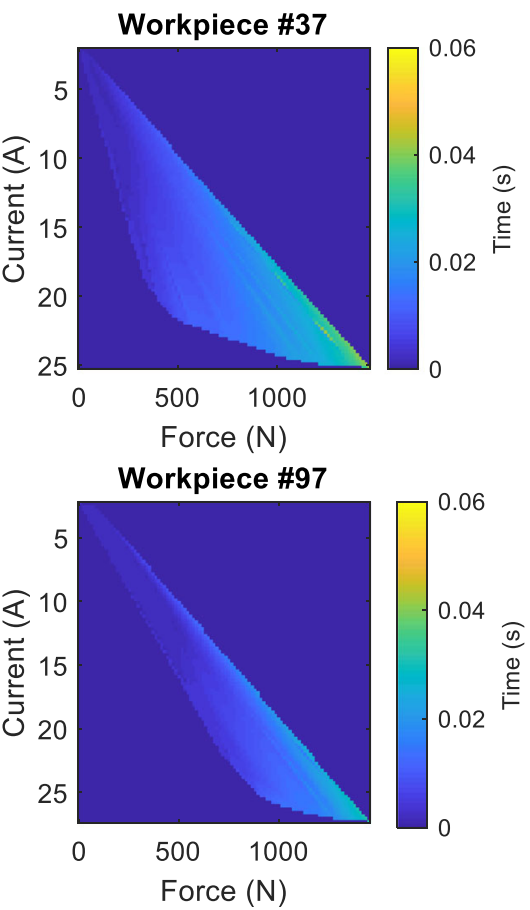
Fig. 10 Raw signals for monitoring the finishing process

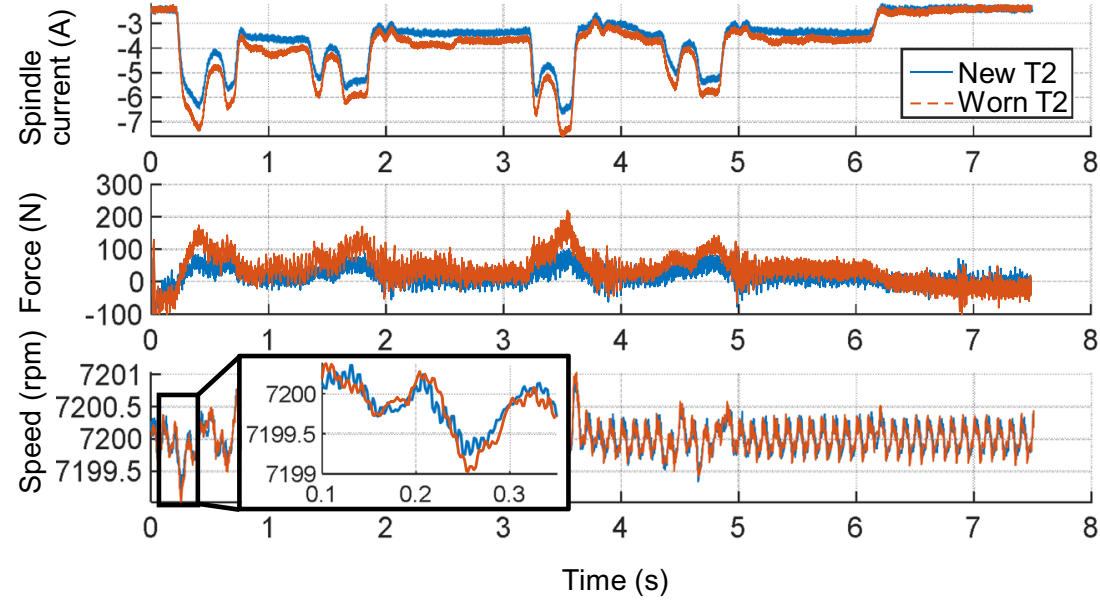

processes are still indispensable. As shown in Fig. 12, regular real measurements were conducted to control the virtual measurement.

Thus, the reliability of the developed tool wear monitoring system can be improved. Negative trends of quality features that are possibly caused by tool wear can thereby be fast identified and serve as decision support for tool changing. Outliers far from normal data points need to be specifically examined on a measurement machine.

Approach 2: Even though the cutting forces of the finishing process are relatively small when compared with those of roughing, it was possible by means of the second method to obtain a tool wear indicator. The cutting force, spindle current, and timestamp signals are used as in the previous process to create a tool wear indicator for the finishing process. Figure 13 shows that the wear profile of the finishing process is similar to that of roughing. However, in the finishing process, it is possible to see that wear occurs more slowly having as parameter the effective area of the color map. In the first pieces in which the tool is new, the effective triangle range is large. With the increase of wear,

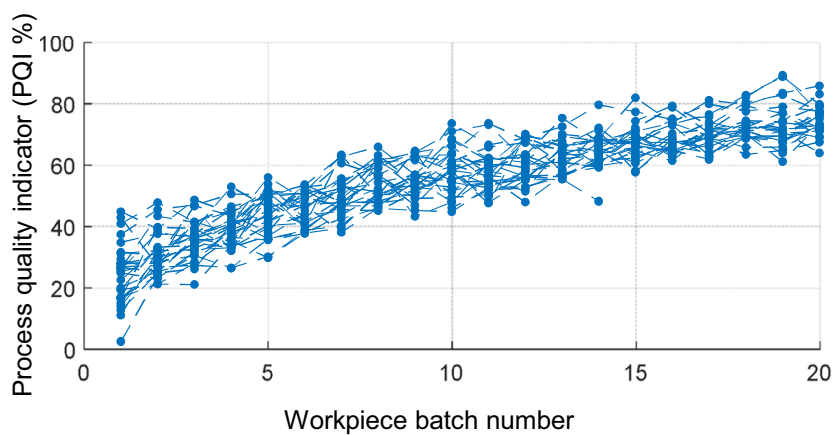

Fig. 11 Process quality indicator for the finishing process (plotted: 600 workpieces and 30 tools) the force and current during the same cutting process shift to the higher values, so that the effective triangle range becomes smaller.

\section{Summary and outlook}

This paper introduces two new methods for quality and tool wear monitoring in production environments in context of the Internet of Production. Both developed methods are based only on machine internal data, which were collected and handled by edge devices that do not influence the CNC performance. High-frequency trace data and datadriven simulation models were combined together to enable a real-time tool monitoring. In the first approach, a process quality indicator has been constructed based on different input signals. This reduced the uncertainties in estimation and improved the robustness of the system. For a workpiece that requires a high surface finish, virtual quality has been taken into account to support the tool monitoring based on process quality indicator. As all the data were collected from real industrial practice, this method shows its good practicability and reliability. To evaluate the applicability of the proposed method to other machining operations, further tests considering different tools and operations need to be carried out. The second approach constructs the wear indicator as a heat map, which succinctly describes the cutting history over time. Considering that the color mapbased methodology presented detailed results, an extension of the methodology using artificial intelligence and deep learning approaches to predict the wear and tear of the tool based on the color map would be a viable and interesting option to explore.

To further increase the applicability and reliability of the method, additional sensors such as spindle-integrated 
Fig. 12 Monitoring of a test series in production

Fig. 13 Color maps representation of tool wear progression for the finishing process
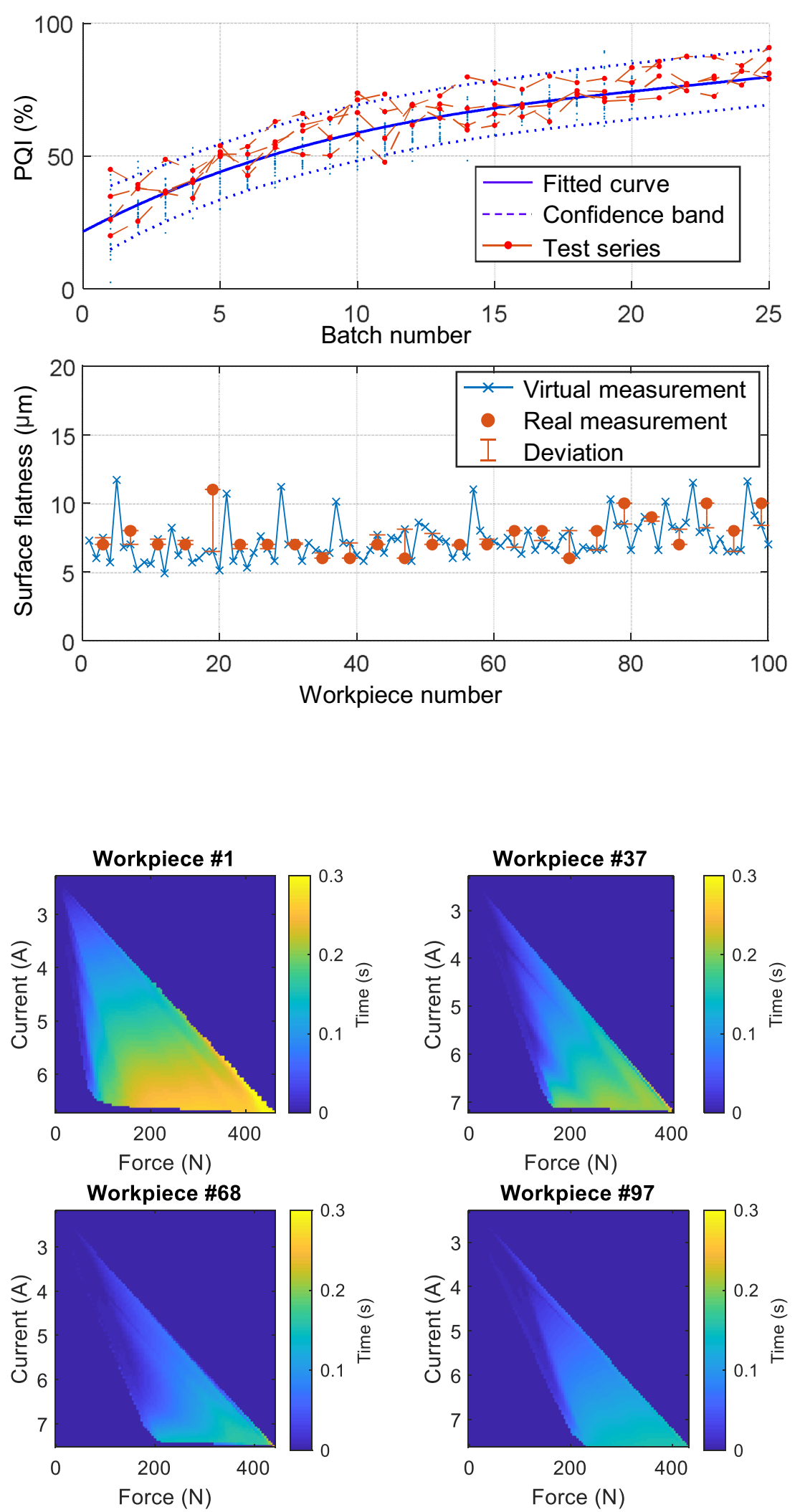
force sensors [27] can be combined with the developed tool monitoring system. By utilizing the force sensors, cutting forces can be accurately measured above $10 \mathrm{kHz}$; thus, the virtual quality measurement and wear estimation can be further improved.

Acknowledgements The authors would like to thank Heidelberger Druckmaschinen AG for supporting this study.

Author contribution This paper introduces a new concept for monitoring tool wear at shop floor level in the context of Industry 4.0. The research was carried out by analyzing high-frequency production data, which were collected automatically during manufacturing. To predict the optimal timing for tool changing, virtual quality of the workpiece was combined with multiple machine internal signals and a force model. The developed method can enable a statistical quality control, give information about whether the process is stable or not, and provide indications whether the tool wear progress will affect the workpiece quality.

Funding Open Access funding enabled and organized by Projekt DEAL. This study is supported by Heidelberger Druckmaschinen AG. Funded by the Deutsche Forschungsgemeinschaft (DFG, German Research Foundation) under Germany's Excellence Strategy - EXC 2023 Internet of Production - 390621612.

\section{Declarations}

Conflict of interest The authors have no competing interests.

Open Access This article is licensed under a Creative Commons Attribution 4.0 International License, which permits use, sharing, adaptation, distribution and reproduction in any medium or format, as long as you give appropriate credit to the original author(s) and the source, provide a link to the Creative Commons licence, and indicate if changes were made. The images or other third party material in this article are included in the article's Creative Commons licence, unless indicated otherwise in a credit line to the material. If material is not included in the article's Creative Commons licence and your intended use is not permitted by statutory regulation or exceeds the permitted use, you will need to obtain permission directly from the copyright holder. To view a copy of this licence, visit http:// creativecommonshorg/licenses/by/4.0/.

\section{References}

1. Schuh G, Prote J, Gützlaff A, Thomas K, Sauermann F, Rodemann $\mathrm{N}$ (2019) Internet of production: rethinking production management. In: Wulfsberg J, Hintze W, Behrens BA (eds) Production at the leading edge of technology. Springer Vieweg, Berlin, pp 533542. https://doi.org/10.1007/978-3-662-60417-5_53

2. Pennekamp J et al (2019) Towards an infrastructure enabling the internet of production. ICPS 2019, 31-37. https://doi.org/10.1109/ ICPHYS.2019.8780276

3. Brecher C, Epple A, Knape S, Schmidt S (2018) Network architecture components for shop floor level (translated form German). Zeitschrift für wirtschaftlichen Fabrikbetrieb (ZWF) 113(5):342-345. https://doi.org/10.3139/104.111904
4. Königs M, Brecher C (2018) Process-parallel virtual quality evaluation for metal cutting in series production. Procedia Manuf 26:1087-1093. https://doi.org/10.1016/j.promfg.2018.07.145

5. Brecher C, Wiesch M, Wellmann F (2019) Productivity Increase - Model-based optimisation of NC-controlled milling processes to reduce machining time and improve process quality. IFAC PapersOnLine 52(13):1803-1807. https://doi.org/10.1016/j.ifacol. 2019.11.463

6. Motorcu AR, Güllü A (2006) Statistical process control in machining, a case study for machine tool capability and process capability. Mater Des 27:364-372. https://doi.org/10.1016/j.matdes.2004. 11.003

7. MacGregor JF, Kourti $\mathrm{T}$ (1995) Statistical process control of multivariate processes. Control Eng Pract 3:403-414. https://doi.org/10.1016/0967-0661(95)00014-L

8. Zhou Y, Xue W (2018) Review of tool condition monitoring methods in milling processes. Int J Adv Manuf Technol 96:25092523. https://doi.org/10.1007/s00170-018-1768-5

9. Jurkovic J, Korosec M, Kopac J (2005) New approach in tool wear measuring technique using CCD vision system. Int J Mach Tool Manuf 45:1023-1030. https://doi.org/10.1016/j.ijmachtools. 2004.11.030

10. Wang M, Wang J (2012) CHMM For tool condition monitoring and remaining useful life prediction. Int $\mathbf{J}$ Adv Manuf Technol 59:463-471. https://doi.org/10.1007/s00170-011-3536-7

11. Bhattacharyya P, Sengupta D, Mukhopadhyay S (2007) Cutting force-based real-time estimation of tool wear in face milling using a combination of signal processing techniques. Mech Syst Signal Process 21:2665-2683. https://doi.org/10.1016/j.ymssp. 2007.01.004

12. Cheng K, Niu ZC, Wang RC et al (2017) Smart cutting tools and smart machining: development approaches, and their implementation and application perspectives. Chin J Mech Eng 30:1162-1176. https://doi.org/10.1007/s10033-017-0183-4

13. Wang C, Cheng K, Minton T, Rakowski R (2014) Development of a novel surface acoustic wave (SAW) based smart cutting tool in machining hybrid dissimilar material. Manufacturing Letters (2)21-25. https://doi.org/10.1016/j.mfglet.2013.12.003

14. Nouri M, Fussell B, Ziniti B, Linder E (2015) Real-time tool wear monitoring in milling using a cutting condition independent method. Int J Mach Tool Manuf 89:1-13. https://doi.org/10.1016/ j.ijmachtools.2014.10.011

15. Choudhury SK, Rath S (2000) In-process tool wear estimation in milling using cutting force model. J Mater Process Technol 99(1):113-119. https://doi.org/10.1016/S0924-0136(99)00396-9

16. Ammouri AH, Hamade RF (2014) Current rise criterion: a process independent method for tool-condition monitoring and prognostics. Int J Adv Manuf Technol 72:509-519. https://doi.org/ 10.1007/s00170-014-5679-9

17. Rizal M, Ghani JA, Nuawi M, Che H (2014) A review of sensor system and application in milling process for tool condition monitoring. Res J Appl Sci Technol 7(10):2083-2097. https://doi.org/10.19026/rjaset.7.502

18. Brecher C, Epple A, Fey M, Königs M, Neus S, Wellmann F (2017) Self-Learning Production Systems (translated from german: Lernende Produktionssysteme). In: Internet of Production für agile Unternehmen: AWK Aachener WerkzeugmaschinenKolloquium 2017, Apprimus Verlag, 135-161. ISBN 978-386359-512-8

19. Wang G, Yang Y, Zhang Y, Xie Q (2014) Vibration sensor based tool condition monitoring using $v$ support vector machine and locality preserving projection. Sens Actuators A Phys 209:24-32. https://doi.org/10.1016/j.sna.2014.01.004

20. Zhang C, Yao X, Zhang J, Jin H (2016) Tool condition monitoring and remaining useful life prognostic based on a 
wireless sensor in dry milling operations. Sensors 16(795):1-20. https://doi.org/10.3390/s16060795

21. Zhang X, Lu X, Wang S, Wang W, Li W (2018) A multi-sensor based online tool condition monitoring system for milling process. Procedia CIRP 72:1136-1141. https://doi.org/10.1016/j.procir. 2018.03.092

22. Huang Z, Zhu J, Lei J, Li X, Tian F (2020) Tool wear predicting based on multi-domain feature fusion by deep convolutional neural network in milling operations. J Intell Manuf 31:953-966. https://doi.org/10.1007/s10845-019-01488-7

23. de Farias A, de Almeida SLR, Delijaicov S, Seriacopi V, Bordinassi E (2020) Simple machine learning allied with datadriven methods for monitoring tool wear in machining processes. Int J Adv Manuf Technol 109:2491-2501. https://doi.org/10.1007/ s00170-020-05785-x
24. Aslan D, Altintas Y (2018) Prediction of cutting forces in 5axis milling using feed drive current measurements. IEEE/ASME Trans Mechatron 23(2):833-844. https://doi.org/10.1109/TME CH.2018.2804859

25. Fey M, Epple A, Kehne S, Brecher C (2016) Method for determining the axle load on linear and rotary axes. Patent DE102016013890A1

26. ISO 16610-22 (2015) Geometrical product specifications (GPS) - Filtration - Part 22: Linear profile filters: Spline filters

27. Brecher C, Eckel H, Motschke T, Fey M, Epple A (2019) Estimation of the virtual workpiece quality by the use of a spindleintegrated process force measurement. CIRP Ann 68:381-384. https://doi.org/10.1016/j.cirp.2019.04.020

Publisher's note Springer Nature remains neutral with regard to jurisdictional claims in published maps and institutional affiliations. 